\title{
Which platelet function test best reflects the in vivo plasma concentrations of ticagrelor and its active metabolite?
}

\section{The HARMONIC study}

\author{
Marek Koziński; Małgorzata Ostrowska; Piotr Adamski'; Joanna Sikora²; Adam Sikora³; Aleksandra Karczmarska-Wódzka²; \\ Michał Piotr Marszałł ; Joanna Boinska4; Ewa Laskowska'; Ewa Obońska²; Tomasz Fabiszak'; Jacek Kubica ${ }^{5}$ \\ ${ }^{1}$ Department of Principles of Clinical Medicine, Collegium Medicum, Nicolaus Copernicus University, Bydgoszcz, Poland; ${ }^{2}$ Department of Pharmacology and Therapy, Collegium \\ Medicum, Nicolaus Copernicus University, Bydgoszcz, Poland; ${ }^{3}$ Department of Medicinal Chemistry, Collegium Medicum, Nicolaus Copernicus University, Bydgoszcz, Poland; \\ ${ }^{4}$ Department of Pathophysiology, Collegium Medicum, Nicolaus Copernicus University, Bydgoszcz, Poland; ${ }^{5}$ Department of Cardiology and Internal Medicine, Collegium Medicum, \\ Nicolaus Copernicus University, Bydgoszcz, Poland
}

\begin{abstract}
Summary
Aim of this study was assessment of the relationship between concentrations of ticagrelor and its active metabolite (AR-C124910XX) and results of selected platelet function tests. In a single-centre, cohort study, patients with myocardial infarction underwent blood sampling following a $180 \mathrm{mg}$ ticagrelor loading dose intake (predose, 1, 2, 3, 4, 6, 12, 24 hours postdose) to perform pharmacokinetic and pharmacodynamic assessments. Platelet reactivity was evaluated using the VASP-assay, the VerifyNow device and the Multiplate analyzer. Analysis of $36 \mathrm{pa}-$ tients revealed high negative correlations between ticagrelor concentrations and platelet reactivity evaluated with all three platelet function tests (the VASP-assay: $\mathrm{RS}=-0.722 ; \mathrm{p}<0.0001$; the VerifyNow device: $\mathrm{RS}=-0.715 ; \mathrm{p}<0.0001$; the Multiplate analyzer: $\mathrm{RS}=-0.722$; $\mathrm{p}<0.0001$ ), with no significant differences between correlation coefficients. Similar results were found for AR-C124910XX. Platelet reactivity values assessed with all three methods generally correlated well with
\end{abstract}

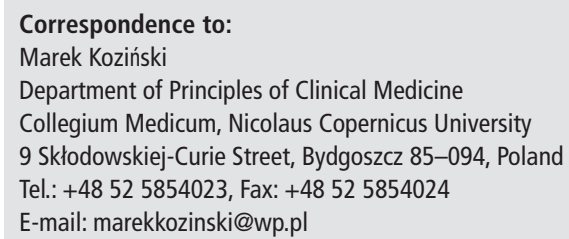

Study registration: ClinicalTrials.gov identifier NCT02690454.

$¥ \quad$ These authors contributed equally to this work. each other; however, a significantly higher correlation $(p<0.02)$ was demonstrated between the VerifyNow and Multiplate tests ( $R S=0.707$; $p<0.0001$ ) than in other assay combinations (the VASP-assay and the VerifyNow device: $R S=0.595 ; p<0.0001$; the VASP-assay and the Multiplate analyzer: $\mathrm{RS}=0.588 ; \mathrm{p}<0.0001)$. With respect to the recognition of high platelet reactivity, we found higher measurement concordance between the VerifyNow and Multiplate tests compared with other assay combinations, while for low platelet reactivity, only results of the VerifyNow and Multiplate assay were related to each other. Platelet reactivity measurements performed with the VASP, VerifyNow and Multiplate tests show comparably strong negative correlations with ticagrelor and AR-C124910XX concentrations.

\section{Keywords}

Ticagrelor, AR-C124910XX, flow cytometric VASP-assay, VerifyNow device, Multiplate analyzer

Financial support:

The HARMONIC study was supported by Collegium Medicum of Nicolaus Copernicus University (NCU CM grant no. 202) and did not receive any external funding.

Received: July 15, 2016

Accepted after minor revision: August 24, 2016

Epub ahead of print: September 15, 2016

http://dx.doi.org/10.1160/TH16-07-0535

Thromb Haemost 2016; 116: 1140-1149

\section{Introduction}

Ticagrelor is a direct-acting, reversible platelet $\mathrm{P} 2 \mathrm{Y}_{12}$ receptor inhibitor recommended by both European and American guidelines as first line therapy in a broad spectrum of patients with acute coronary syndromes (ACS) $(1,2)$. The faster, stronger and more consistent inhibition of platelet function by ticagrelor and its active metabolite (AR-C124910XX) translated into improved patient outcomes in the landmark PLATO trial $(3,4)$. Importantly, multiple platelet function tests are usually simultaneously used in pharmacodynamic studies on $\mathrm{P}_{2} \mathrm{Y}_{12}$ receptor inhibitors. Additionally, platelet function testing is a tool which may aid in better clinical decision making (5). According to both European and American ex- perts, there are three recommended platelet function tests, namely the VerifyNow device, the Multiplate analyzer and the vasodilatorstimulated phosphoprotein (VASP) phosphorylation assay, and none of these three methods is preferred over another (6-8).

Notably, due to the reversibility of the receptor binding as well as fast onset and offset of action, plasma concentrations of ticagrelor and AR-C124910XX well reflect the extent of platelet P2 $\mathrm{Y}_{12}$ receptor inhibition $(3,9)$. So far there are no studies linking measurements of ticagrelor and AR-C124910XX concentrations with comparative evaluation of methods used for platelet reactivity assessment.

The primary goal of this trial was to evaluate the relationship between plasma concentrations of ticagrelor and AR-C124910XX 
on the one hand and the results of all three recommended platelet function tests on the other in patients with acute myocardial infarction (AMI). The secondary study goal included assessment of correlation and agreement between the results of the three compared platelet function tests.

\section{Methods}

\section{Study design}

The study was designed as a prospective, single-centre, investigator-initiated, cohort trial. Consecutive AMI patients admitted to The Department of Cardiology and Internal Medicine between 6:00 a.m. and 6:00 p.m. received orally a $300 \mathrm{mg}$ loading dose (LD) of plain aspirin (Polpharma SA, Starogard Gdański, Poland) and were screened with respect to their eligibility for the study enrollment. Each patient provided written informed consent for participation prior to any study specific procedures. Inclusion criteria were as follows: age between 18 and 80 years, male or non-pregnant female patients with a diagnosis of ST-segment elevation AMI (STEMI) or non-ST-segment elevation AMI (NSTEMI), and provision of informed consent for angiography and percutaneous coronary intervention (PCI). Exclusion criteria were: treatment with any $\mathrm{P}_{2} \mathrm{Y}_{12}$ receptor inhibitor within 14 days prior to the study enrollment, ongoing treatment with oral anticoagulant or chronic therapy with low-molecular-weight heparin, active bleeding, Killip class III or IV during screening for eligibility, respiratory failure, and history of coagulation disorders (10). Additionally, we prespecified that patients receiving a glycoprotein (GP) IIb/IIIa receptor inhibitor will be excluded from the primary analysis as this therapy may affect the results of platelet reactivity assessment with the VerifyNow and Multiplate tests. STEMI and NSTEMI were diagnosed in line with The Third Universal Definition of Myocardial Infarction (11). All study participants received orally a 180 mg LD of ticagrelor with $250 \mathrm{ml}$ of tap water immediately after baseline blood sampling. Subsequently, within 15 minutes (min) from the ticagrelor $\mathrm{LD}$, all patients underwent a coronary angiography assessment followed by PCI, if necessary. The study participants were given a $90 \mathrm{mg}$ ticagrelor maintenance dose every 12 hours (h) and a $75 \mathrm{mg}$ aspirin maintenance dose every $24 \mathrm{~h}$ following the LDs. The study was approved by the Local Ethics Committee in accordance with the Helsinki Declaration.
Table 1: Baseline characteristics of study participants. Data are shown as median (interquartile range) or number (\%). AMI - acute myocardial infarction; CABG - coronary artery bypass grafting; n/a - not applicable; NSTEMI - nonST-segment elevation myocadial infarction; $\mathrm{PCI}-$ percutaneous coronary intervention; STEMI ST-segment elevation myocardial infarction.

\begin{tabular}{|c|c|c|c|c|}
\hline Variable & $\begin{array}{l}\text { Overall study } \\
\text { population } \\
(n=45)\end{array}$ & $\begin{array}{l}\text { Patients included } \\
\text { in the primary } \\
\text { analysis } \\
(n=36)\end{array}$ & $\begin{array}{l}\text { Patients not } \\
\text { included in the } \\
\text { primary analysis } \\
(n=9)\end{array}$ & P-value \\
\hline Age [years] & $62.0(55.0-72.0)$ & $62.0(55.5-73.5)$ & $59.0(55.0-67.0)$ & 0.477 \\
\hline Female & $11(24.4)$ & $8(22.2)$ & $3(33.3)$ & 0.666 \\
\hline Body mass index $\left[\mathrm{kg} / \mathrm{m}^{2}\right]$ & $26.9(24.8-30.1)$ & $26.7(24.6-30.2)$ & $26.9(26.5-28.4)$ & 0.650 \\
\hline STEMI/NSTEMI & $31(68.9) / 14(31.1)$ & $22(61.1) / 14(38.9)$ & $9(100) / 0(0)$ & 0.024 \\
\hline Hypertension & $20(44.4)$ & $18(50)$ & $2(22.2)$ & 0.261 \\
\hline Diabetes mellitus & $9(20)$ & $8(22.2)$ & $1(11.1)$ & 0.661 \\
\hline Dyslipidaemia & $40(88.9)$ & $31(86.1)$ & $9(100)$ & $\mathrm{n} / \mathrm{a}$ \\
\hline Current smoker & $19(42.2)$ & $15(41.7)$ & $4(44.4)$ & 1.00 \\
\hline Prior AMI & $7(15.6)$ & 7 (19.4) & 0 & $\mathrm{n} / \mathrm{a}$ \\
\hline Prior $\mathrm{PCl}$ & $8(17.8)$ & $8(22.2)$ & 0 & $\mathrm{n} / \mathrm{a}$ \\
\hline Prior $C A B G$ & 0 & 0 & 0 & $\mathrm{n} / \mathrm{a}$ \\
\hline $\begin{array}{l}\text { Prior non-severe heart } \\
\text { failure }\end{array}$ & $1(2.2)$ & $1(2.8)$ & 0 & $\mathrm{n} / \mathrm{a}$ \\
\hline $\begin{array}{l}\text { Prior non-haemorrhagic } \\
\text { stroke }\end{array}$ & 0 & 0 & 0 & $\mathrm{n} / \mathrm{a}$ \\
\hline $\begin{array}{l}\text { Peripheral arterial } \\
\text { disease }\end{array}$ & $2(4.4)$ & $2(5.6)$ & 0 & $\mathrm{n} / \mathrm{a}$ \\
\hline Chronic renal disease & $2(4.4)$ & $2(5.6)$ & 0 & $\mathrm{n} / \mathrm{a}$ \\
\hline $\begin{array}{l}\text { Chronic obstructive } \\
\text { pulmonary disease }\end{array}$ & 0 & 0 & 0 & $\mathrm{n} / \mathrm{a}$ \\
\hline Gout & $3(6.6)$ & $2(5.6)$ & $1(11.1)$ & 0.500 \\
\hline $\begin{array}{l}\text { Morphine use in the } \\
\text { acute phase of AMI }\end{array}$ & $21(46.7)$ & 15 (41.7) & $6(66.7)$ & 0.267 \\
\hline
\end{tabular}




\section{Study endpoints}

Blood samples were collected at multiple time points in order to assess study endpoints in a broad range of ticagrelor/AR-C124910XX concentrations and values of platelet reactivity. For each study participant, eight values of ticagrelor/AR-C124910XX concentrations and platelet reactivity, if not stated otherwise, were included in the final analysis. Values of correlation coefficients or intra-class correlation coefficients were compared between the assessed platelet function tests.

The primary study endpoint was the correlation coefficient between ticagrelor concentrations and platelet reactivity measurements, while the secondary study endpoints included:

- correlation coefficient between AR-C124910XX concentrations and platelet reactivity measurements,

- correlation coefficient between ticagrelor concentrations and inhibition of platelet reactivity (inhibition of platelet reactivity was defined as pre-LD of ticagrelor platelet reactivity minus actual platelet reactivity, divided by pre-LD of ticagrelor platelet reactivity; for each study participant, seven values of ticagrelor concentration and inhibition of platelet reactivity (for sampling points at $1,2,3,4,6,12,24$ h post-LD) were included in the final analysis),

- correlation coefficient between AR-C124910XX concentrations and inhibition of platelet reactivity (additional description same as above),

- Bland-Altman analysis assessing agreement between the standardised values of platelet reactivity measured with the compared platelet function tests (standardized platelet reactivity was defined as actual platelet reactivity minus mean platelet reactivity, divided by standardised deviation of platelet reactivity),

- correlation coefficient between platelet reactivity measured with the compared platelet function tests,
- intra-class correlation coefficient between platelet reactivity measured with the compared platelet function tests,

- $\kappa$ statistic to assess agreement between platelet reactivity measurements with the compared platelet function tests in terms of identifying patients with high platelet reactivity (HPR) or low platelet reactivity (LPR).

Additionally, we decided to perform an exploratory, post-hoc subanalysis comparing STEMI and NSTEMI patients.

\section{Blood sampling}

Blood samples were collected using a venous catheter (18G) inserted into a forearm vein at eight pre-defined time points (prior to the $\mathrm{LD}$ of ticagrelor and 1, 2, 3, 4, 6, 12 and $24 \mathrm{~h}$ post-LD). The first 3-5 $\mathrm{ml}$ of blood were discarded to avoid spontaneous platelet activation.

\section{Measurement of ticagrelor and AR-C124910XX concentrations}

Ticagrelor and AR-C124910XX plasma concentrations were measured separately using liquid chromatography coupled with tandem mass spectrometry as described in our previous publication (12). Lower limits of quantification were $4.69 \mathrm{ng} / \mathrm{ml}$ both for ticagrelor and AR-C124910XX.

\section{Assessment of platelet reactivity}

Platelet reactivity was evaluated using the flow cytometric VASPassay (VASP; BioCytex, Marseille, France), the VerifyNow device (VerifyNow P2Y12 assay, Accumetrics, Inc., San Diego, CA, USA) and multiple electrode aggregometry (MEA) with the Multiplate analyzer (ADPtest, Roche Diagnostics International Ltd.,

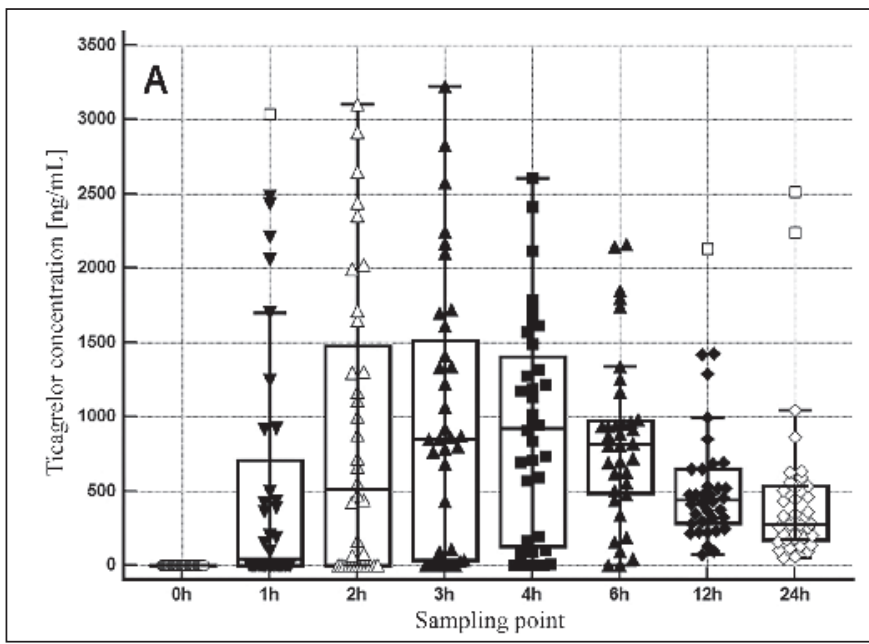

Figure 1: Ticagrelor (A) and AR-C124910XX (B) over time in 36 study participants. Drug concentrations were measured at baseline, and at 1, 2, 3, $4,6,12$, and $24 \mathrm{~h}$ after administration of a $180 \mathrm{mg}$ ticagrelor loading dose

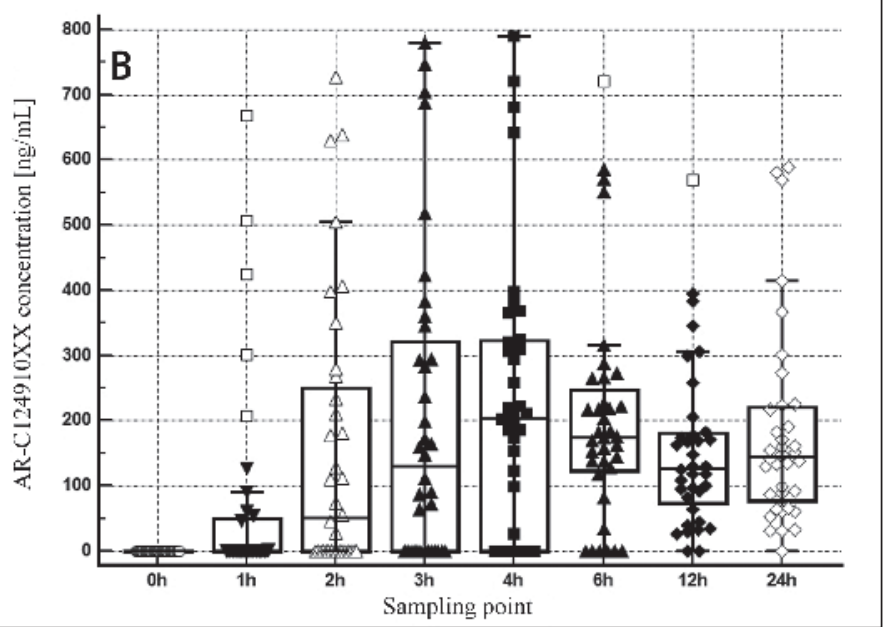

followed by a $90 \mathrm{mg}$ ticagrelor maintenance dose every $12 \mathrm{~h}$. Boxes and whiskers represent medians, interquartile ranges and non-outlier ranges. Extreme outliers are plotted using empty squares. 


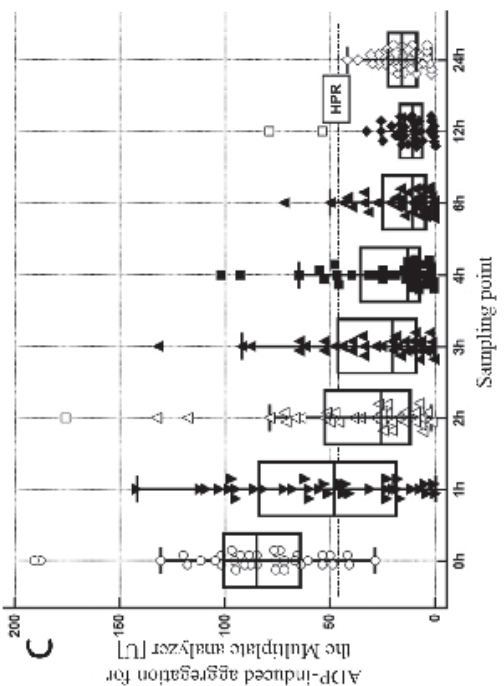

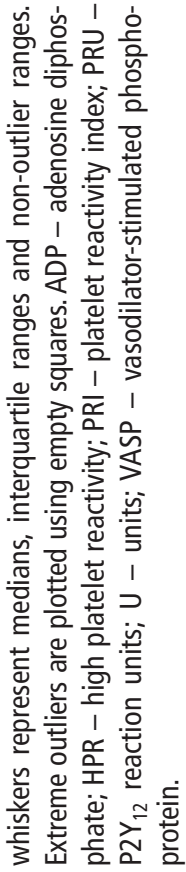
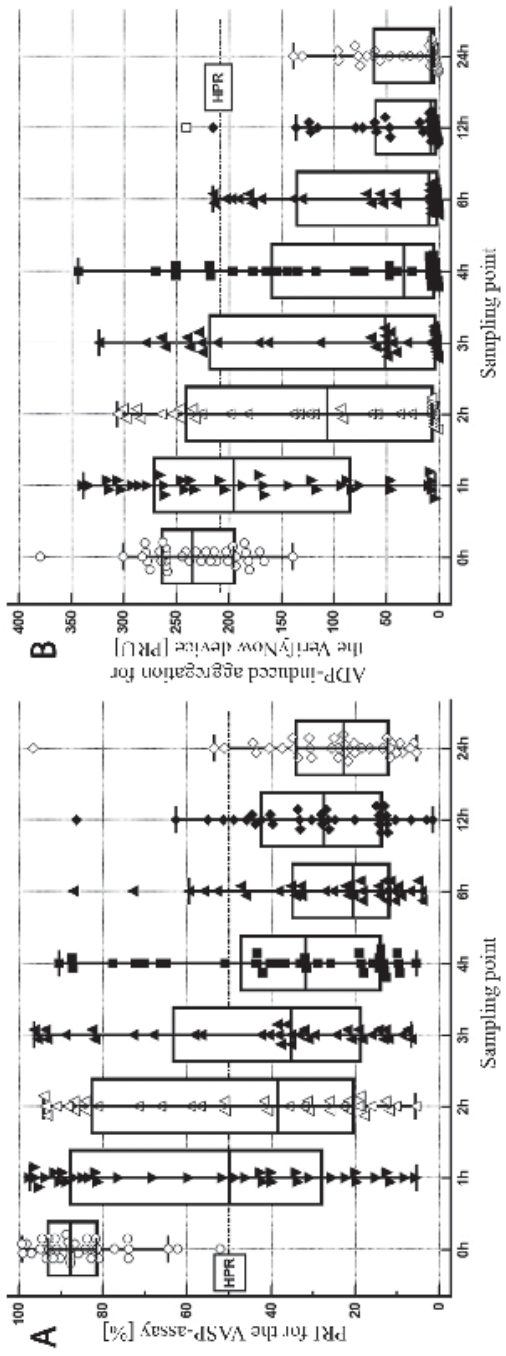

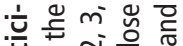

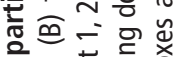

京范然

홀

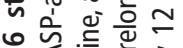

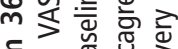

$\therefore$.

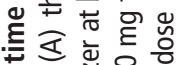

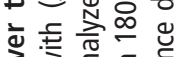

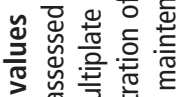

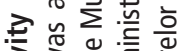

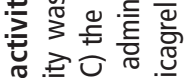

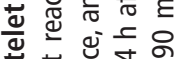

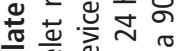

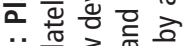

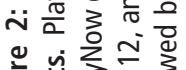

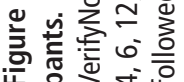

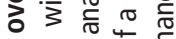

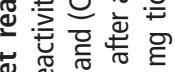

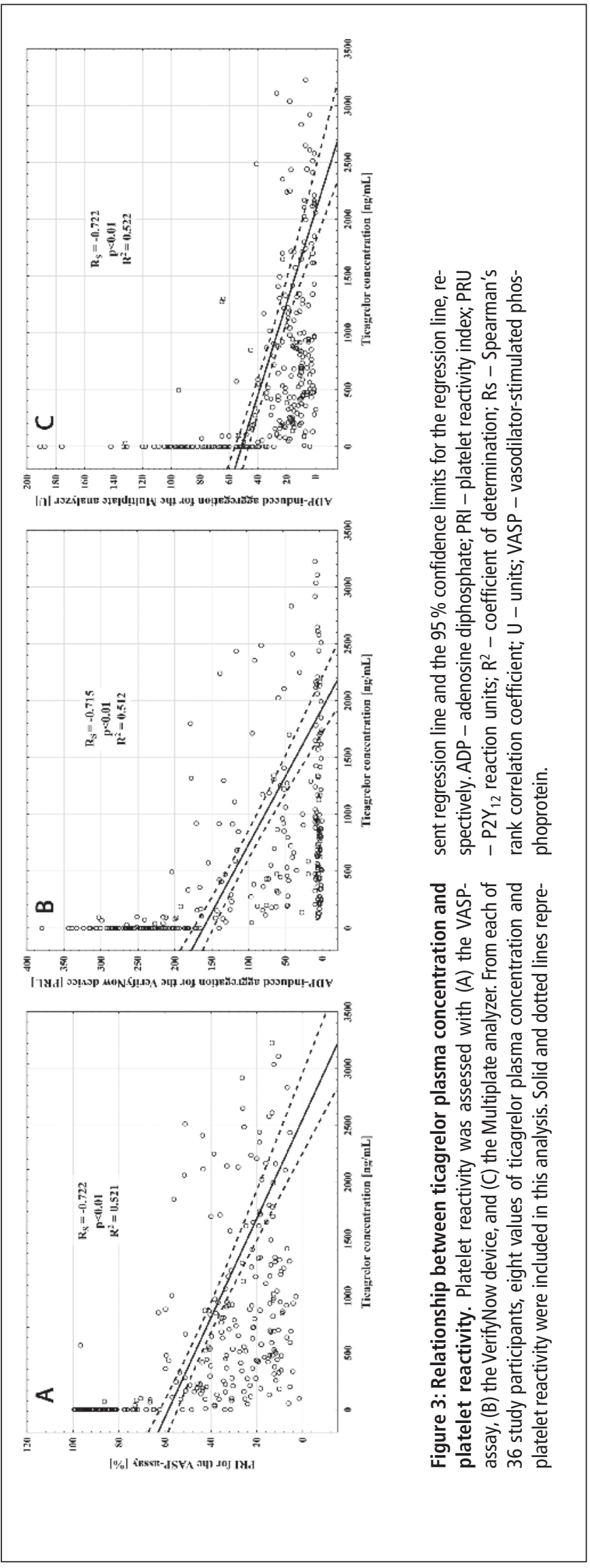



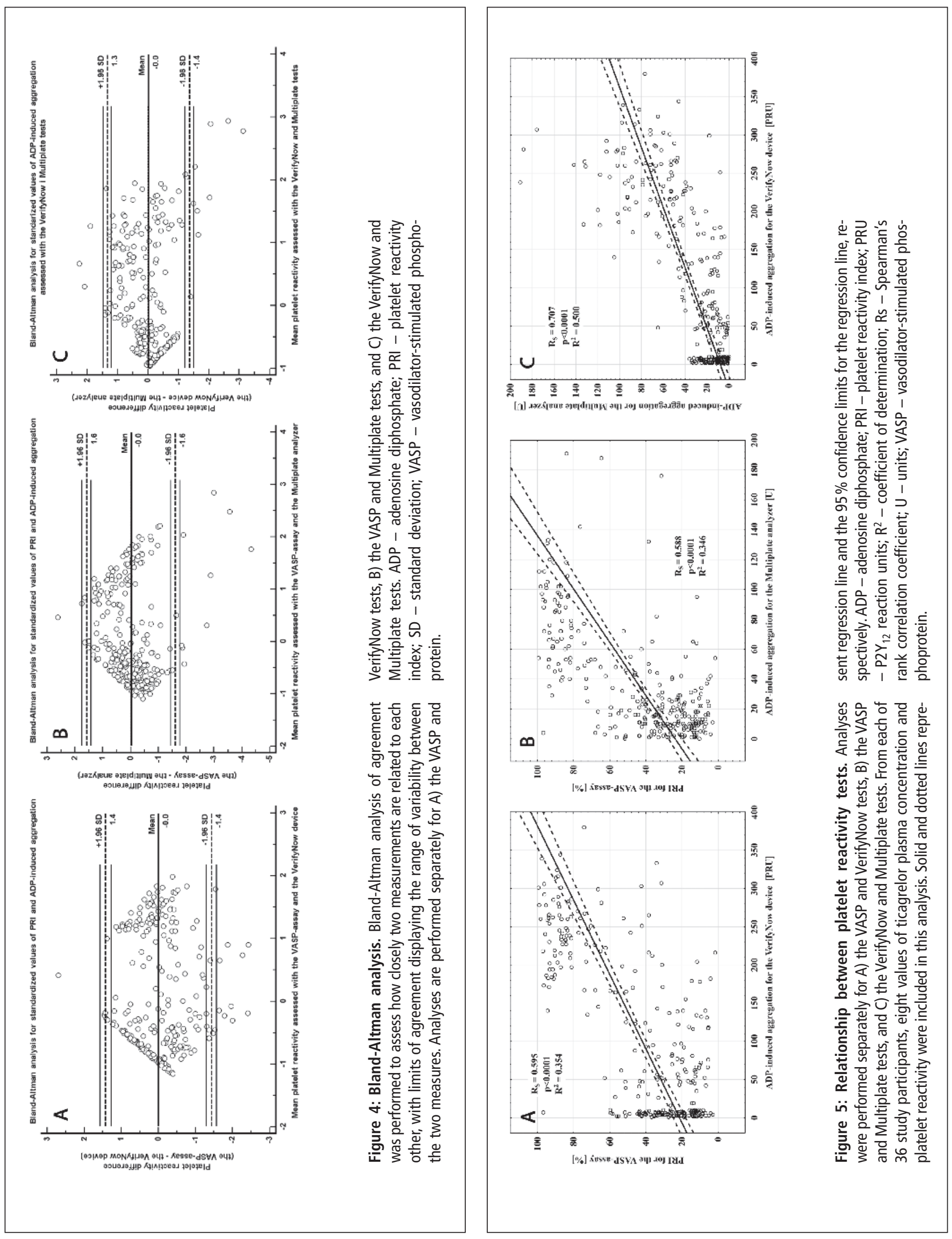
Rotkreuz, Switzerland). All the above methods were described in detail in our previous publications $(12,13)$. Platelet reactivity was expressed as platelet reactivity index (PRI), $\mathrm{P}_{2} \mathrm{Y}_{12}$ reaction units (PRU), and area under the aggregation curve (AUC) for the VASPassay, the VerifyNow device and the Multiplate analyzer, respectively. HPR was defined as PRI $>50 \%$, AUC $>46$ units (U), and PRU $>208$, when assessed with the VASP, Multiplate and VerifyNow tests, respectively (6-8). LPR was identified if AUC was $<19 \mathrm{U}$ for the Multiplate analyzer, while due to inconsistencies between expert consensus documents two different cut-off values for LPR were used, for the VASP-assay $(<10 /<16 \%)$ and for the VerifyNow device $(<85 /<95$ PRU) (6-8).

\section{Sample size calculation and statistical analysis}

Due to the lack of an appropriate reference study, we decided to perform an internal pilot study on an initial 15 participants to estimate the final sample size for the primary study endpoint. Spearman's correlation coefficient between ticagrelor concentrations and platelet reactivity measured with the VASP-assay was considered a reference for our calculations as this test is regarded as the most accurate tool assessing the effect of thienopyridine or non-thienopyridine inhibitors on the platelet $\mathrm{P} 2 \mathrm{Y}_{12}$ receptor. A value of -0.777 was reached for the first 15 recruited patients. We estimated that a sample size of 35 subjects would have a $95 \%$ power to detect a $20 \%$ difference in Spearman's correlation coefficient between our reference and values obtained for either the VerifyNow device or the Multiplate analyzer, with a 0.05 two-sided significance level. Values of the lowest detectable differences in
Spearman's correlation coefficient were $-0.622(80 \% \mathrm{x}-0.777)$ and -0.932 (120\%x-0.777).

The Shapiro-Wilk test showed a lack of normal distribution for the majority of the analysed quantitative variables, including concentrations of ticagrelor and AR-C124910XX and values of platelet reactivity. Therefore these variables were presented as medians and interquartile ranges (IQR). An extreme outlier was defined as a value that was smaller than the lower quartile minus tripled IQR, or larger than the upper quartile plus tripled IQR. In figures, these values were plotted using a different marker. Depending on the presence or absence of normal distribution, intergroup comparisons were performed with the Student's t-test for independent samples or the Mann-Whitney U test. Unpaired categorical variables were compared using the $\mathrm{Chi}^{2}$ test, with Yates' correction if required, or by Fisher's exact test. Correlations were tested with the Spearman's rank correlation test. Coefficients of determination, which are squared correlation coefficients $\left(\mathrm{R}^{2}\right)$, were also provided as they indicate how well data fit a statistical model. Additionally, intra-class correlation coefficients were calculated. Correlation coefficients were compared using dedicated tests. Agreement between assays to evaluate standardised platelet reactivity was assessed through the Bland-Altman analysis. The $\kappa$ statistic was used to investigate agreement among the assays in identification of patients with HPR or LPR. A value of two sided $p<0.05$ was regarded as statistically significant. There were no missing values in our data. The statistical analysis and sample size calculation were carried out using the Statistica 12.0 (StatSoft, Tulsa, OK, USA), MedCalc 15.4 (MedCalc Software, Mariakerke, Belgium) and PQStat 1.6.0.544 (PQStat Software, Poznań/Plewiska, Poland) packages.

Table 2: Agreement between platelet reactivity tests to identify high platelet reactivity as assessed by the $\mathrm{K}$ statistic. Values in the table refer to numbers of measurements, if not stated otherwise. HPR - high platelet reactivity; PRI - platelet reactivity index; PRU - P2Y ${ }_{12}$ reaction units; $U$ - units.

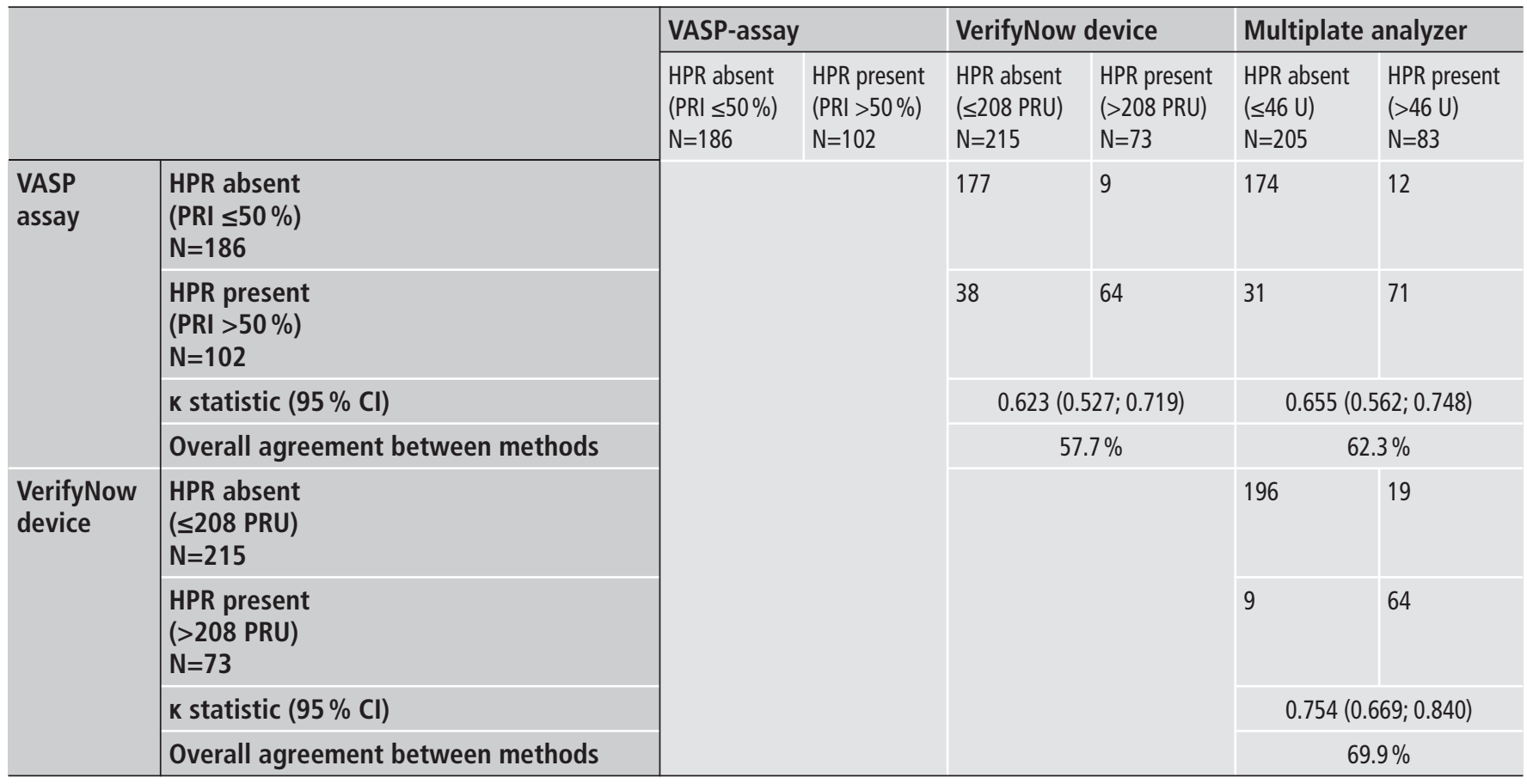


Table 3: Agreement between platelet reactivity tests to identify low platelet reactivity as assessed by the $\mathrm{K}$ statistic. Values in the table refer to numbers of measurements, if not stated otherwise. LPR - low platelet reactivity; PRI - platelet reactivity index; PRU - P2Y 12 reaction units; U - units.

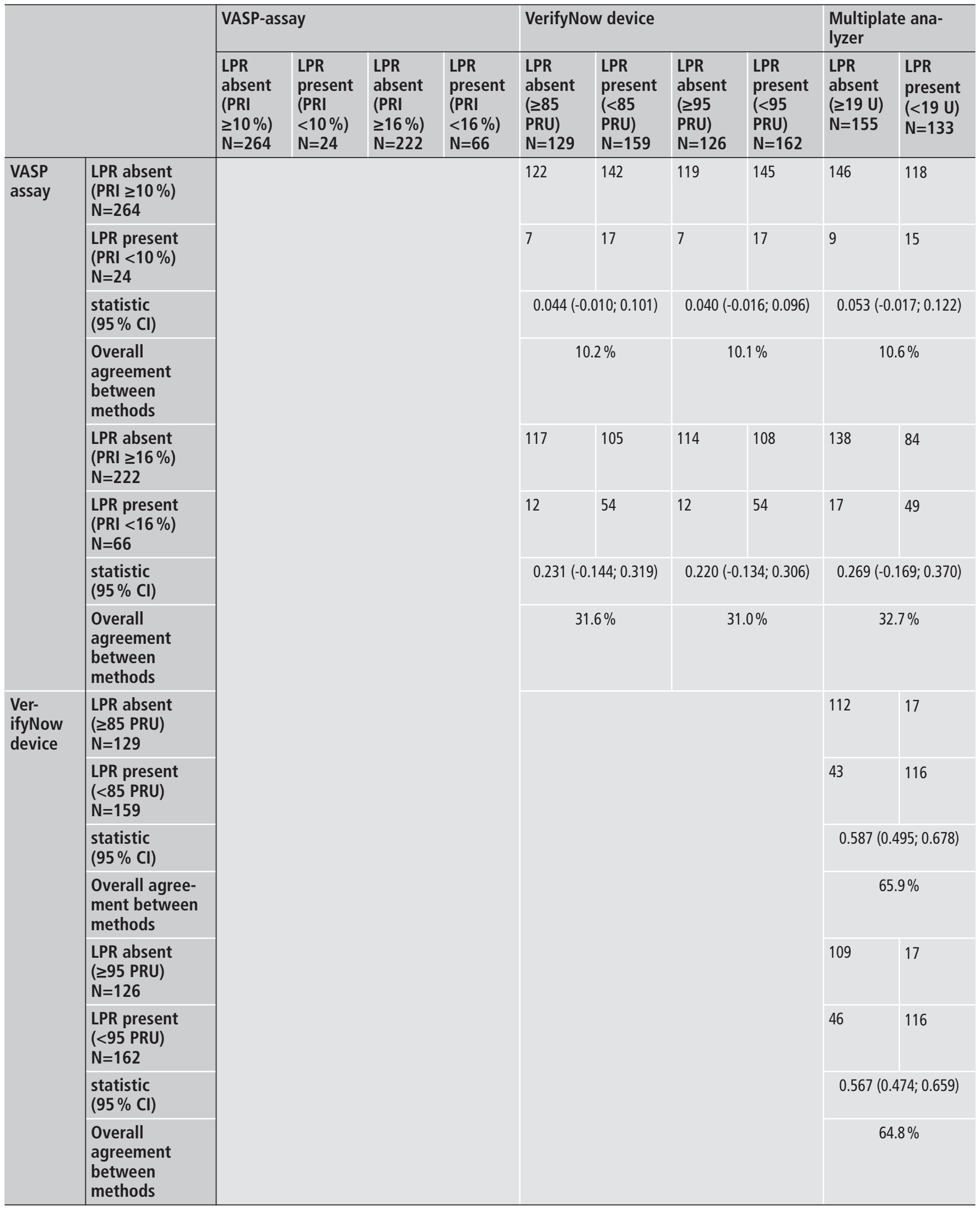




\section{Results Study population}

Between November 2015 and March 2016, 45 AMI patients were enrolled into the study. Baseline characteristics of the study population are presented in $>$ Table 1. During PCI, nine patients received a GP IIb/IIIa receptor inhibitor and were excluded from the primary analysis. Therefore, pharmacokinetic and pharmacodynamic assessments were performed in 36 patients.

\section{Ticagrelor and AR-C124910XX concentrations and platelet reactivity in the analysed patients}

We found a considerable interindividual variability in terms of ticagrelor and AR-C124910XX concentrations and platelet reactivity. Median concentrations of ticagrelor and AR-C124910XX were highest $4 \mathrm{~h}$ after the administration of the LD of ticagrelor ( $>$ Figure 1). Administration of the $180 \mathrm{mg}$ ticagrelor LD substantially decreased platelet reactivity, which was on average close to the cutoff value for HPR at the $1 \mathrm{~h}$ sampling point and reached lowest values between 6 and $24 \mathrm{~h}$ after the ticagrelor LD, depending on the method applied ( $>$ Figure 2).

\section{Relationship between concentrations of ticagrelor and AR-C124910XX and the results of assessed platelet function tests}

We observed high negative correlations between ticagrelor concentrations and platelet reactivity evaluated with all three platelet function tests ( Figure 3), with no significant differences between correlation coefficients. Concordant results were found for AR-C124910XX (Suppl. Figure 1, available online at www.throm bosis-online.com), also with no significant differences between correlation coefficients. Additionally, both ticagrelor and AR-C124910XX concentrations positively correlated with the inhibition of platelet reactivity by ticagrelor, but $\mathrm{R}^{2}$ values were generally lower than for correlations with platelet reactivity (Suppl. Figures 2 and 3, available online at www.thrombosis-online.com). Consistent with the above results, we failed to find any differences between correlation coefficients determined for the compared methods of platelet reactivity assessment.

\section{Agreement between platelet reactivity tests}

The Bland-Altman analysis revealed good agreement between the standardized values of platelet reactivity when measured with all compared platelet function tests ( Figure 4). Importantly, approximately $95 \%$ of differences between the standardised values of platelet reactivity in all three comparisons were between the limits of agreement on the Bland-Altman plots.

Platelet reactivity values assessed with all three methods generally correlated well with each other ( Figure 5); however, a significantly higher correlation $(\mathrm{p}<0.02)$ was demonstrated between the VerifyNow and Multiplate tests than in other assay combinations. Similar results were obtained for intra-class correlation coefficients between platelet reactivity when measured with the compared platelet function tests (the VASP-assay and the VerifyNow device: ICC $=0.737$; $\mathrm{p}<0.000001$; the VASP-assay and the Multiplate analyzer: $\mathrm{ICC}=0.671$; $\mathrm{p}<0.000001$; the VerifyNow device and the Multiplate analyzer: ICC $=0.762 ; \mathrm{p}<0.000001)$, however the difference reached statistical significance only in the comparison between extreme values of intraclass correlation coefficients $(\mathrm{p}<0.02)$.

Interestingly, although the $\kappa$ statistic demonstrated relatively good agreement between all three compared platelet function tests in terms of HPR identification, it was highest between the VerifyNow device and the Multiplate analyzer ( Table 2). On the other hand, for LPR, only results for the VerifyNow and Multiplate test were related to each other ( $>$ Table 3$)$.

\section{Comparison of STEMI and NSTEMI patients}

Ticagrelor and AR-C124910XX concentrations and values of platelet reactivity over time in STEMI $(\mathrm{n}=22)$ versus NSTEMI $(\mathrm{n}=14)$ patients are displayed in Suppl. Figures 4 and 5 (available online at www.thrombosis-online.com). Notably, both STEMI and NSTEMI subjects showed considerable inter-individual variability in terms of ticagrelor and AR-C124910XX concentrations and platelet reactivity at all sampling points. Importantly, in STEMI when compared with NSTEMI, we found lower maximum ticagrelor concentration (1167.92 (804.51; 1615.21) $\mathrm{ng} / \mathrm{ml}$ vs $2342.68(1299.24 ; 2511.59) \mathrm{ng} / \mathrm{ml} ; \mathrm{p}<0.004)$ and lower maximum AR-C124910XX concentration (251.97 (175.49; $316.86) \mathrm{ng} / \mathrm{ml}$ vs $402.85(272.55 ; 638.38) \mathrm{ng} / \mathrm{ml} ; \mathrm{p}<0.02)$, longer time to achieve these two (ticagrelor: $5(3 ; 12) \mathrm{h}$ vs $2(1 ; 4) \mathrm{h}$; p<0.01; AR-C124910XX: $6(4 ; 12)$ h vs $3,5(3 ; 4) h ; p=0.082)$, as well as delayed maximum antiplatelet effect according to all three methods of platelet function assessment.

\section{Discussion}

We believe, this is the first study which demonstrates that all three recommended platelet function tests similarly reflect the in vivo plasma concentrations of ticagrelor and its active metabolite. This observation was independently confirmed in two distinct analyses when correlations between drug concentrations and platelet reactivity or platelet inhibition were assessed. Additionally, values of platelet reactivity determined by all three methods generally correlated well with each other; however, a significantly higher correlation was demonstrated between aggregation-based assays, i.e. the VerifyNow and Multiplate tests, than in combinations containing the VASP assay. Importantly, further findings include: i) a relatively good agreement between the recommended platelet function tests in terms of HPR identification, again with the highest measurement concordance being noted between the VerifyNow and Multiplate tests, and ii) a lack of agreement between the compared methods in terms of LPR recognition, except for considerable agreement between the VerifyNow and Multiplate assays. Importantly, our results are largely applicable since ticagrelor is a widely used drug. 
Our results suggest that multiple platelet function tests may be no more required in future clinical trials evaluating the antiplatelet effect of ticagrelor. Depending of their local availability, easiness of the use, costs, concomitant therapy with GP IIb/IIIa receptor inhibitors, investigator's preferences, or other factors, one of the tests investigated in our study may be selected for pharmacodynamic studies. We expect that our observations will allow to simplify the design of future studies and lead to resource savings. On the other hand, the rates of on-ticagrelor HPR and particularly LPR determined using currently recommended cut-off values considerably differed between particular platelet function tests in our study and this issue warrants further investigation.

In clopidogrel-treated ACS patients undergoing PCI, HPR and LPR are considered to be risk factors for ischaemic complications, predominantly stent thrombosis, and bleeding events, respectively $(5,14-16)$. Although some evidence suggests that LPR on ticagrelor is associated with increased risk of bleeding, there is no solid rationale for platelet function testing in patients on ticagrelor therapy (17-19). Apart from superior pharmacokinetic and pharmacodynamic properties of ticagrelor in comparison to clopidogrel, major randomised trials on personalised antiplatelet therapy failed to demonstrate clinical benefits of this approach (20-22).

Previous studies investigating the relationship between clopidogrel active metabolite concentrations and the results of platelet function testing generally favoured the VASP-assay (23-26). Bou-

\section{What is known about this topic?}

- Ticagrelor is a direct-acting, reversible platelet $\mathrm{P}_{2} \mathrm{Y}_{12}$ receptor inhibitor recommended by both European and American guidelines in patients with acute coronary syndromes. Ticagrelor and its active metabolite (AR-C124910XX) inhibit platelet function faster, more strongly and more consistently than clopidogrel, the former standard of care.

- Multiple platelet function tests are usually simultaneously used in pharmacodynamic studies on $\mathrm{P}_{2} \mathrm{Y}_{12}$ receptor inhibitors. According to both European and American experts, there are three recommended platelet function tests, namely the VerifyNow device, the Multiplate analyzer and the VASP assay and none of these three methods is preferred over another.

- So far there are no studies linking measurements of ticagrelor and AR-C124910XX concentrations with comparative evaluation of methods used for platelet reactivity assessment.

\section{What does this paper add?}

- This is the first study which demonstrates that all three recommended platelet function tests similarly reflect the in vivo plasma concentrations of ticagrelor and its active metabolite.

- Our results suggest that multiple platelet function tests may be no more required in future clinical trials evaluating the antiplatelet effect of ticagrelor.

- Additionally, our study indicates a delayed and weakened ticagrelor effect in the acute phase of STEMI vs. NSTEMI. man et al. found that inhibition of platelet reactivity determined with the VASP-assay $\left(\mathrm{R}^{2}=0.56 ; \mathrm{p}<0.001\right)$ when compared with the VerifyNow device $\left(\mathrm{R}^{2}=0.48 ; \mathrm{p}<0.001\right)$ and light transmission aggregometry (LTA) with $20 \mu \mathrm{mol} / \mathrm{l}$ adenosine diphosphate - ADP $\left(\mathrm{R}^{2}=0.46 ; \mathrm{p}=0.001\right.$ for peak and $\mathrm{R}^{2}=0.47 ; \mathrm{p}<0.001$ for late inhibition of platelet reactivity) showed best correlation with peak plasma concentration of the clopidogrel active metabolite (23). Liang et al. also demonstrated superiority of the VASP-assay $(\mathrm{R}=-0.58)$ over LTA $(\mathrm{R}=-0.47)$ and the Multiplate analyzer $(\mathrm{R}=-0.34)$ in terms of correlation with log-transformed concentrations of clopidogrel active metabolite ( $\mathrm{p}$ for all correlation coefficients <0.01) (24). Additionally, Delavenne et al. observed a higher correlation coefficient between the total area under the clopidogrel active metabolite plasma concentration-time curve from time zero to infinity and inhibition of platelet reactivity for the VASP-assay $\left(\mathrm{R}^{2}=0.72 ; \mathrm{p}=0.008\right)$ than for LTA $(10 \mu \mathrm{mol} / \mathrm{l} \mathrm{ADP}$ : $\left.\mathrm{R}^{2}=0.49 ; \mathrm{p}<0.05 ; 5 \mu \mathrm{mol} / 1 \mathrm{ADP}: \mathrm{R}^{2}=0.61 ; \mathrm{p}<0.05\right)$ (25). Importantly, clopidogrel active metabolite is prone to rapid inactivation and irreversibly inhibits platelet function. These facts should be taken into account when interpreting the findings of the above studies.

Our study indicates a delayed and weakened ticagrelor effect in the acute phase of STEMI vs. NSTEMI. However, due to the fact that these findings are derived from an exploratory, post hoc subanalysis, they warrant in-depth investigation in prospective, powered studies, which are ongoing $(26,27)$. Importantly, some previous reports indicated that the antiplatelet action of ticagrelor in the STEMI setting may be delayed, but they lacked pharmacokinetic assessment and subjects with other presentations of coronary artery disease as a control group $(28,29)$. Future studies should particularly address the underlying mechanisms of impaired ticagrelor antiplatelet effect in the STEMI setting and explore its clinical relevance.

Several limitations of our study should be acknowledged. Firstly, only AMI patients admitted to hospital during daytime were enrolled in the study due to difficulties with frequent blood sampling and processing at night. Secondly, patients treated with GP IIb/IIIa receptor inhibitors were excluded from the primary analysis. Thirdly, although we investigated all recommended methods of platelet function assessment (6-8), several other, less validated, assays are also available. Fourthly, despite the fact that HPR and LPR in patients undergoing PCI remain well-documented predictors of ischaemic and bleeding complications, studies linking ticagrelor and AR-C124910XX concentrations with clinical events are lacking. Finally, the study was underpowered for assessment of clinical endpoints, for sub-group analyses and for detection of mild differences between correlation coefficients.

In conclusion, platelet reactivity measurements performed with the VASP-assay, the VerifyNow device and the Multiplate analyzer show comparably strong negative correlations with ticagrelor and AR-C124910XX concentrations. With respect to the recognition of HPR, our study indicates higher measurement concordance between the VerifyNow and Multiplate tests than with other assay combinations, while for LPR, only results of the VerifyNow and Multiplate assays are related to each other. 


\section{Acknowledgements}

R. Bilski, E. Kolasińska, J.M. Kubica, P. Lisiecka, K. Obońska, N. Skibińska, P. Sobczak, W. Sroka, K. Stankowska and P. Szarwas are acknowledged for their assistance in the processing of blood samples.

\section{Conflicts of interest}

Dr Marek Koziński received honoraria for lectures from AstraZeneca. Dr Jacek Kubica received a consulting fee from AstraZeneca. All other authors have reported that they have no relationships relevant to the contents of this paper to disclose.

\section{References}

1. Roffi M, Patrono C, Collet JP, et al. 2015 ESC Guidelines for the management of acute coronary syndromes in patients presenting without persistent ST-segment elevation. Eur Heart J 2016; 37: 267-315.

2. Steg PG, James SK, Atar D, et al. ESC Guidelines for the management of acute myocardial infarction in patients presenting with ST-segment elevation. Eur Heart J 2012; 33: 2569-2619.

3. Gurbel PA, Bliden KP, Butler K, et al. Randomized double-blind assessment of the ONSET and OFFSET of the antiplatelet effects of ticagrelor versus clopidogrel in patients with stable coronary artery disease: the ONSET/OFFSET study. Circulation 2009; 120: 2577-2585

4. Wallentin L, Becker RC, Budaj A, et al. Ticagrelor versus clopidogrel in patients with acute coronary syndromes. N Engl J Med 2009; 361: 1045-1057.

5. Aradi D, Kirtane A, Bonello L, et al. Bleeding and stent thrombosis on P2Y12-inhibitors: collaborative analysis on the role of platelet reactivity for risk stratification after percutaneous coronary intervention. Eur Heart J 2015; 36: 1762-1771.

6. Aradi D, Storey RF, Komócsi A, et al. Expert position paper on the role of platelet function testing in patients undergoing percutaneous coronary intervention. Eur Heart J 2014; 35: 209-215.

7. Aradi D, Collet JP, Mair J, et al. Platelet function testing in acute cardiac care - is there a role for prediction or prevention of stent thrombosis and bleeding? Thromb Haemost 2015; 113: 221-230.

8. Tantry US, Bonello L, Aradi D, et al. Consensus and update on the definition of on-treatment platelet reactivity to adenosine diphosphate associated with ischemia and bleeding. J Am Coll Cardiol 2013; 62: 2261-2273.

9. Storey RF, Angiolillo DJ, Bonaca MP, et al. Platelet inhibition with ticagrelor 60 $\mathrm{mg}$ versus $90 \mathrm{mg}$ twice daily in the PEGASUS-TIMI 54 Trial. J Am Coll Cardiol 2016; 67: 1145-1154.

10. Which platelet function test best reflects the in vivo plasma concentrations of ticagrelor and its active metabolite? The HARMONIC study. ClinicalTrials.gov Identifier: NCT02690454 https://clinicaltrials.gov/ct2/show/ NCT02690454?term=NCT02690454\&rank=1. Accessed August 16, 2016.

11. Thygesen K, Alpert JS, Jaffe AS, et al; Writing Group on the Joint ESC/ACCF/ AHA/WHF Task Force for the Universal Definition of Myocardial Infarction. Third universal definition of myocardial infarction. Eur Heart J 2012; 33: 2551-2567.

12. Kubica J, Adamski P, Ostrowska M, et al. Morphine delays and attenuates ticagrelor exposure and action in patients with myocardial infarction: the randomized, double-blind, placebo-controlled IMPRESSION trial. Eur Heart J 2016; 37: 245-252.
13. Koziński M, Obońska K, Stankowska K, et al. Prasugrel overcomes high onclopidogrel platelet reactivity in the acute phase of acute coronary syndrome and maintains its antiplatelet potency at 30-day follow-up. Cardiol J 2014; 21: $547-556$.

14. Mangiacapra F, Patti G, Barbato E, et al. A therapeutic window for platelet reactivity for patients undergoing elective percutaneous coronary intervention: results of the ARMYDA-PROVE (Antiplatelet therapy for Reduction of MYocardial Damage during Angioplasty-Platelet Reactivity for Outcome Validation Effort) study. JACC Cardiovasc Interv 2012; 5: 281-289.

15. Bonello L, Mancini J, Pansieri M, et al.; Relationship between post-treatment platelet reactivity and ischemic and bleeding events at 1-year follow-up in patients receiving prasugrel. J Thromb Haemost 2012; 10: 1999-2005.

16. Stone GW, Witzenbichler B, Weisz G, et al. ADAPT-DES Investigators. Platelet reactivity and clinical outcomes after coronary artery implantation of drug-eluting stents (ADAPT-DES): a prospective multicentre registry study. Lancet 2013; 382: 614-623.

17. Siller-Matula JM, Hintermeier A, Kastner J, et al. Distribution of clinical events across platelet aggregation values in all-comers treated with prasugrel and ticagrelor. Vascul Pharmacol 2016; 79: 6-10.

18. Alexopoulos D, Stavrou K, Koniari I, et al. Ticagrelor vs prasugrel one-month maintenance therapy: impact on platelet reactivity and bleeding events. Thromb Haemost 2014; 112: 551-557.

19. Windecker S, Kolh P, Alfonso F, et al. 2014 ESC/EACTS Guidelines on myocardial revascularization. Eur Heart J 2014; 35: 2541-2619.

20. Price MJ, Berger PB, Teirstein PS, et al. Standard- vs high-dose clopidogrel based on platelet function testing after percutaneous coronary intervention: the GRAVITAS randomized trial. J Am Med Assoc 2011; 305: 1097-1105.

21. Trenk D, Stone GW, Gawaz M, et al. A randomized trial of prasugrel versus clopidogrel in patients with high platelet reactivity on clopidogrel after elective percutaneous coronary intervention with implantation of drug-eluting stents: results of the TRIGGER-PCI (Testing Platelet Reactivity In Patients Undergoing Elective Stent Placement on Clopidogrel to Guide Alternative Therapy With Prasugrel) study. J Am Coll Cardiol 2012; 59: 2159-2164.

22. Collet JP, Cuisset T, Rangé G, et al. Bedside monitoring to adjust antiplatelet therapy for coronary stenting. N Engl J Med 2012; 367: 2100-2109.

23. Bouman HJ, Parlak E, van Werkum JW, et al. Which platelet function test is suitable to monitor clopidogrel responsiveness? A pharmacokinetic analysis on the active metabolite of clopidogrel. J Thromb Haemost 2010; 8: 482-488.

24. Liang Y, Johnston M, Hirsh J, et al. Relation between clopidogrel active metabolite levels and different platelet aggregation methods in patients receiving clopidogrel and aspirin. J Thromb Thrombolysis 2012; 34: 429-436.

25. Delavenne X, Mallouk N, Piot M, et al. Is there really a relationship between the plasma concentration of the active metabolite of clopidogrel and the results of platelet function tests? J Thromb Haemost 2010; 8: 2334-2338.

26. Pharmacokinetics and pharmacodynamics of ticagrelor in patients with stable angina, NSTEMI and STEMI undergoing PCI. ClinicalTrials.gov Identifier: NCT02012140 https://clinicaltrials.gov/ct2/show/NCT02012140?term= NCT02012140 \&rank=1.Accessed August 16, 2016.

27. Comparison of ticagrelor pharmacokinetics and pharmacodynamics in STEMI and NSTEMI patients (PINPOINT). ClinicalTrials.gov Identifier: NCT02602444 https://clinicaltrials.gov/ct2/show/NCT02602444?term= NCT02602444\&rank=1. Accessed August 16, 2016.

28. Alexopoulos D, Xanthopoulou I, Gkizas V, et al. Randomized assessment of ticagrelor versus prasugrel antiplatelet effects in patients with ST-segment-elevation myocardial infarction. Circ Cardiovasc Interv 2012; 5: 797-804.

29. Parodi G, Valenti R, Bellandi B, et al. Comparison of prasugrel and ticagrelor loading doses in ST-segment elevation myocardial infarction patients: RAPID (Rapid Activity of Platelet Inhibitor Drugs) primary PCI study. J Am Coll Cardiol 2013; 61: 1601-1606. 